\section{APLICACIONES DEL CEMENTO REFORZADO CON FIBRA DE VIDRIO (GRC)}

\author{
Angel Barros Llerena \\ Ingeniero Industrial \\ Cementos y Fibras, S. A. \\ Juan Manuel Caballero \\ Arquitecto Técnico \\ Hispano-Alemana de Construcciones, S. A. \\ Víctor Zaldo \\ Ing. Téc, de Construcciones Civiles \\ Hispano-Alemana de Construcciones, S. A.
}

\section{RESUMEN}

El presente artículo tiene como objeto dar a conocer la utilización de fibras de vidrio como refuerzo de los cementos. Se da una breve reseña histórica, se mencionan sus características y comportamiento. Además se presenta un caso práctico y reciente de utilización en la fabricación de elementos de fachada del Estadio «Santiago Bernabéu» del Real Madrid C. de F., y se completa con una relación de los actuales y futuros usos del G.R.C. (glass reinforced cement), denominación inglesa del material más comúnmente utilizado.

\section{Reseña histórica}

El bajo costo, la fácil disponibilidad y capacidad para adaptarse a diferentes procesos de fabricam ción ha hecho que los materiales basados en los conglomerantes hidráulicos hayan tenido una utillzación creciente a lo largo de la historia.

Desafortunadamente para el hombre, los morteros $y$ hormigones en masa son muy resistentes a compresión, pero débiles en tracción, lo que planteó muchas limitaciones en su utilización hasta la aparición del hormigón armado.
Paralelamente al desarrollo de la técnica anterior se realizaron intentos para reforzar los morteros y hormigones con tibras de muy diversos tipos, tales como pelo animal, fibra de sisal, cáñamo, celulo sa, etc. de las cuales, hasta la fecha, únicamente han generado una tecnologia concreta y documentada:

- las fibras de amianto;

- las fibras metálicas;

- la fibra de vidrio resistente a los álcalis Cem-FIL AR.

Desde la aparición de las fibras de vidrio "E", empleadas en el refuerzo de los plásticos, y esperanzados por sus buenas caracteristicas mecánicas se hicieron ensayos para utilizarlas en el refuerzo de cementos experimentándose, en el año 1964 por el Dr. Biryukovick, con cementos aluminosos y siderúrgicos.

Sin embargo, cuando se quisieron utilizar en el refuerzo del cemento portland, que es el más bam rato y disponible de todos los cementos, se presentaron grandes problemas por estar sujetas las fibras al ataque alcalino del cemento hidratado.

En 1967 el Dr. Majumdar, del Building Research Establishment, comenzó a investigar unos vidrios con alto contenido en circonio que demostraron un excelente potencial de resistencia al ataque alcalino del cemento portland.

Tomando como base los vidrios descubiertos por el Dr. Majumdar se estableció, en el año 1968, un plan de investigación para los siguientes diez anos que permitiera conocer perfectamente el comportamiento de este material. La investigación se realizó en estrecha colaboración entre el Building Research Establishment y la compañia inglesa lider mundial en la fabricación del vidrio Pilkington Brothers Limited. Ya en el año 1971 se contaba con:

- Una producción industrial de fibra de vidrio con alto contenido en circonio. La fibra se denominó con el nombre comercial fibra de vidrio Cem-FIL AR. 
- Diferentes sistemas de incorporación de la fim bra de vidrio al cemento, aptos para la fabrica ción de elementos muy diversos.

- Conocimiento muy completo del comporta. miento reológico del G.R.C. así como curvas de variación de resistencia, que permitieron establecer con suficiente fiabllidad sistemas de cálculo y tensiones admisibles de diseño (Fim gura 1).

- Tambien en el año 1971 se llevaron a cabo dos importantes obras con paneles de G.R.C. en un bloque de apartamentos y en el cerramiento de una factoria.

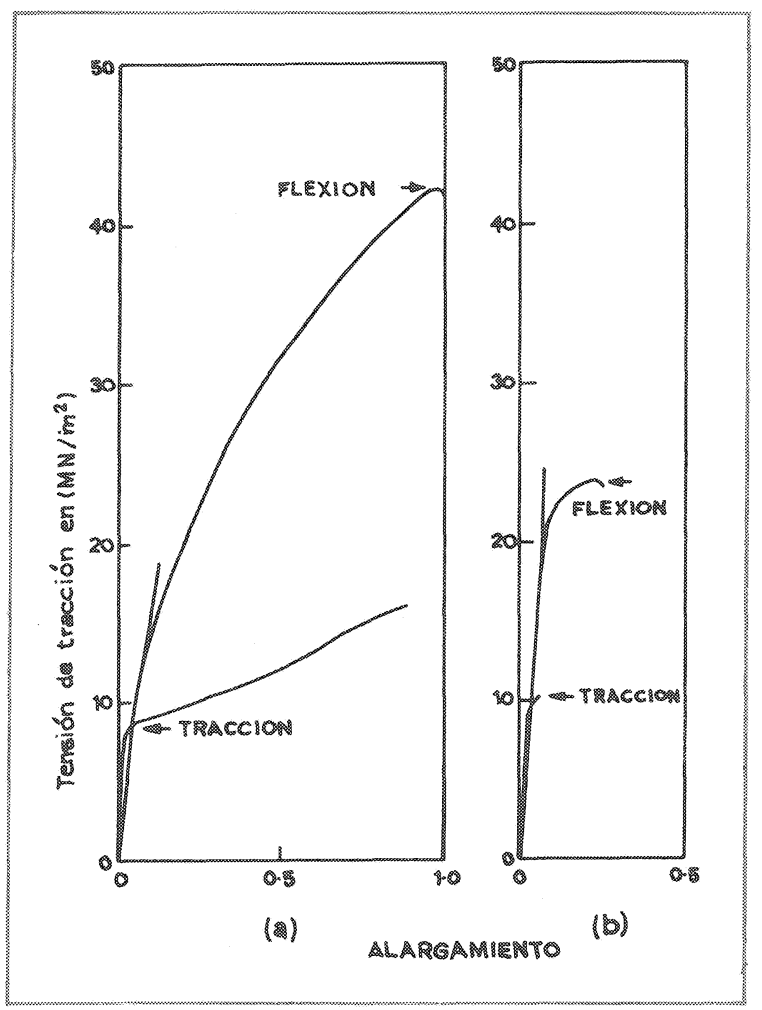

Fig. 1.-Gráfico de tensión-deformación del G.R.C. (a) 28 dias de edad almacenado en agua entre 18 y $20^{\circ} \mathrm{C}$. (b) 5 años de edad almacenado en agua entre 18 y $20^{\circ} \mathrm{C}$.

En el año 1976 el Building Research Establishment publicó el documento CP $3 / 76$ donde se analizaban con todo detalle las propiedades del G.R.C. y se daban unas resistencias estimadas de su comportamiento en un clima medio.

Posteriormente, en el año 1979 el B.R.E. publicó el IP $36 / 79$, documento en el que se analizan los resultados obtenidos en 5 años de pruebas y se dan los valores medios de resistencia esperada a 20 años de edad.

\begin{tabular}{|c|c|c|c|}
\hline Propiedad & Aire & Ague & Intemperie \\
\hline $\begin{array}{l}\text { a) Flexión } \\
\left.\text { Rotur\& (MN/m } / \mathrm{m}^{2}\right) \\
\text { Limite de proporcionalidad }\end{array}$ & $\begin{array}{l}26 \cdot 34 \\
14-16\end{array}$ & $\begin{array}{l}15-20 \\
15-18\end{array}$ & $\begin{array}{l}13-17 \\
13-16\end{array}$ \\
\hline $\begin{array}{l}\text { b) Tracción } \\
\left.\text { Rotura (MN } / \mathrm{m}^{2}\right) \\
\text { Limite de proporcionalidad }\end{array}$ & $\begin{array}{c}11-15 \\
7-8\end{array}$ & $\begin{array}{l}6.8 \\
6.8\end{array}$ & $\begin{array}{l}5-7 \\
5-7\end{array}$ \\
\hline Mádulo de Young $\left(\mathrm{GN} / \mathrm{m}^{2}\right)$ & $22-23$ & 28.34 & 25.32 \\
\hline Resistencina al Impacto(Nimm/mon & $14-20$ & $2 \cdot 3$ & $2 \cdot 4$ \\
\hline
\end{tabular}

Fig. 2. - Propiedades medias de resistencia del G.R.C. fabricado por provección/succión con un $5 \%$ en peso de fibra Cem.FIL ldespués de 20 años/.

En los últimos años la investigación realizada por Pilkington Brothers sobre la fibra Cem-FIL ha pro gresado enormemente, habiéndose obtenido un método de ensayo acelerado que permite estable cer con suficiente garantía las resistencias esperadas a 50 años, en cualquier condición de temperatura y humedad (Fig. 2).

La tecnologia sobre el G.R.C. referente a sis. temas de fabricación, dosificación, comportamiento en diferentes situaciones, incluso en extremas condiciones de uso, así como métodos de control de calidad del proceso de labricación y de los productos acabados ha alcanzado tan amplio desarrollo que hoy dia no cabe considerar el G.R.C. como un solo material sino que, en un sentido más amplio, hay que hablar de una familia que puede adaptar, dentro de ciertos limites, sus ca. racteristicas mecánicas o físicas al fin destinado.

\section{Composición}

Los constituyentes básicos del G.R.C. son: ce mento, arena y agua que se mezclan para formar un mortero al que se añade por diferentes sistemas fibra cortada, de caracteristicas y longitudes adecuadas al producto que se va a fabricar.

Los cementos que se utilizan pueden ser cualquier tipo de los especificados en las normas vigentes.

Las arenas son preferiblemente siliceas de granulometría fina.

El agua debe cumplir lo especificado en la normativa vigente para su empleo en morteros $u$ hor migones.

La fibra de vidrio deberá ser siempre resistente a los álcalis, es decir, la denominada Cem-FIL AR. Normalmente se utiliza en longitudes que oscilan entre los 12 y $38 \mathrm{~mm}$. 


\section{Sistema de fabricación}

Los procesos empleados en la fabricación se han desarrollado a partir de las tecnologias de los plásticos reforzados con fibra de vidrio y del hor. migón prefabricado.

De la industria de los plásticos se ha adoptado el método llamado proyección simultánea, que consiste en: proyectar desde toberas independientes un chorro de mortero pulverizado con aire a presión y otro de fibra de vidrio cortada con hebras de longitud prefijada. El G.R.C., colocado asi en el molde, se puede compactar con rodillo o por un proceso de vacio cuando se están fabricando placas planas.

De la Industria del hormigón se ha adoptado el proceso de colado y compactación por vibración, técnica que se emplea cuando se procesan morteros a los que previamente se les ha adicionado la fibra.

En la práctica, el proceso de proyección ha resultado ser tan extraordinariamente versátil que prácticamente se emplea en la fabricación del $90 \%$ de los productos.

Este proceso se ha visto impulsado también por la facllidad para aplicar capas muy finas de G.R.C., de 2 o $3 \mathrm{~mm}$ con una distribución homogénea de la fibra.

Además permite compactar cuidadosamente las zonas más solicitadas del elemento, incrementar los espesores localmente sin complicar los moldes o adoptar rigidizadores, sin grandes inconvenientes de fabricación.

Con los materiales y procesos brevemente descrim tos se fabrican productos en los que el contenido de libra de vidrio Cem-FIL AR expresado en peso del material fresco recién colocado varia entre el 2,5 y $5 \%$.

- El $2,5 \%$ se emplea para enlucidos resistentes o simplemente impermeables.

- Para procesos de vibración es frecuente emplear un $3,5 \%$ y para proyección es normal el $5 \%$.

\section{Características}

En la resistencia mecánica del G.R.C. influyen los siguientes factores:
1. Contenido, longitud, orientación y diámetro de las libras de vidrio.

2. Proporciones de cemento, arena, agua, y aire de la matriz.

3. El método de fabricación empleado.

4. Condiciones de curado.

5. Edad.

6. Condiciones de servicio, especialmente humedad y temperatura.

La combinación de estos seis factores conforma las propiedades del material, teniendo una particular importancia la edad y condiciones de servicio.

En la Fig. 3 se dan los valores típicos para un G.R.C. con 28 dias de edad curado en agua.

Respecto a las demás caracteristicas físicas que definen el producto tenemos los siguientes valores:

Densidad: Oscila, según el grado de compacidad alcanzado, entre 1,7 y $2,2 \mathrm{t} / \mathrm{m}^{3}$. Es frecuente considerar $2 \mathrm{t} / \mathrm{m}^{3}$.

\begin{tabular}{|c|c|c|c|}
\hline Propiedad & $\begin{array}{l}\text { GRC hecho por pro } \\
\text { yección/succión }\end{array}$ & $\begin{array}{l}\text { GRC necho por pro. } \\
\text { yscción }\end{array}$ & $\begin{array}{l}\text { Premezcla de mex. } \\
\text { cia de alto esfuer. } \\
\text { zo cortante. }\end{array}$ \\
\hline $\begin{array}{l}\text { Densidad } \\
\mathrm{Tn}_{\mathrm{n}} / \mathrm{m}^{2}\end{array}$ & $2.0-2.2$ & $4.7-2.1$ & $1.9-2.1$ \\
\hline \multicolumn{4}{|l|}{$\begin{array}{l}\text { Resistencia } \\
\text { al impacto }\end{array}$} \\
\hline $\mathrm{Nmm} / \mathrm{mm}^{2}$ & $15-30$ & 10.25 & $10-16$ \\
\hline \multicolumn{4}{|l|}{$\begin{array}{l}\text { Resistencia } \\
\text { a La compresión }\end{array}$} \\
\hline $\mathrm{N} / \mathrm{mm}^{2}$ & $60 \cdot 100$ & $50-80$ & \\
\hline \multicolumn{4}{|l|}{$\begin{array}{l}\text { Módulo de } \\
\text { Young }\end{array}$} \\
\hline $\mathrm{RN} / \mathrm{mm}^{2}$ & $15-25$ & $10-20$ & $16-22$ \\
\hline \multicolumn{4}{|l|}{$\begin{array}{l}\text { Tracción en } \\
\text { flexión: } \\
\mathrm{N} / \mathrm{mm}^{2}\end{array}$} \\
\hline $\begin{array}{l}\text { Lumite do propor- } \\
\text { cionsilidad }\end{array}$ & $10 \cdot 16$ & $7-11$ & $8-12$ \\
\hline Rotura & $28-42$ & 21.31 & $18 \cdot 22$ \\
\hline \multicolumn{4}{|l|}{$\begin{array}{l}\text { Tracción } \\
\text { directa } \\
N / \mathrm{mm}^{2}\end{array}$} \\
\hline $\begin{array}{l}\text { Límite da propor- } \\
\text { cionslidsd }\end{array}$ & $7-9$ & $5-7$ & \\
\hline Roture & $10 \cdot 17$ & $8-11$ & \\
\hline $\begin{array}{l}\text { Deformación } \\
\text { La rotura \% } \%\end{array}$ & $0.7 \sim 1.3$ & $0.6-1.2$ & \\
\hline \multicolumn{4}{|l|}{$\begin{array}{l}\text { Esfuerzo corm } \\
\text { t:mte: } \\
N / \mathrm{mm}^{2}\end{array}$} \\
\hline Intarlaminar & $2 \cdot 3$ & $1.5-3$ & \\
\hline de plano & $8-11$ & $7 \cdot 10$ & \\
\hline $\begin{array}{l}\text { Par punzo- } \\
\text { namiento }\end{array}$ & $35-50$ & $30 \cdot 40$ & \\
\hline
\end{tabular}

Fig. 3. - Valores tipicos del G.R.C. curado en agua durante 28 dias. 
Retracción.-Como cualquier material de base cementosa, el G.R.C. está sometido a la retracción, alcanzando valores comprendidos entre 1,5 y 0,6 milimetros/metro.

En piezas de fachada y para climas normales la máxima retracción es inferior a $1 \mathrm{~mm} / \mathrm{m}$.

Fluencia.-Se presenta al igual que en los hormigones y morteros, si bien, tiene menos importancia que los movimientos de retracción por el bajo nivel de tensiones utilizado.

Permeabilidad al aire.-Depende de las condicio nes de curado oscilando entre 3 perms para el G.R.C. mantenido en ambiente seco a 0,15 perms cuando se ha curado en ambiente húmedo.

Permeabilidad al agua.-El G.R.C. cumple las nor mas ISO para un material impermeable.

Dilatación térmica - El coeficiente oscila entre 7 a $12 \times 10^{-6}{ }^{\circ} \mathrm{C}$.

Conductividad térmica. - Varia con el contenido de agua (humedad) y es similar al del hormigón, oscila entre $0,4-0,8 \mathrm{kcal} / \mathrm{m}^{2}{ }^{\circ} \mathrm{C}$.

Aislamiento acústico.-Gracias a su compacidad, origina reducciones de unos 30 decibelios para frecuencias comprendidas entre 350 y $4.000 \mathrm{~Hz}$.

\section{Ventajas de utilización}

El uso del G.R.C. en la construcción presenta las siguientes ventajas:

1. Su mayor relación resistencia/peso permite utilizar espesores pequeños que originan reducciones en peso de hasta un $85 \%$ frente a paneles de hormigón.

2. Facilidad para fabricar por proyección formas complicadas.

3. Gran resistencia al impacto, especialmente en edades tempranas, que facilita considerablemente el manejo de los producios.

4. Combinarse fácilmente con otros materiales de núcleos para la formación de los elementos sandwich.

5. Cortarse o taladrarse fácilmente con herramientas simples, lo que permite resolver incidencias en obra por causas no previstas en el diseño.
6. Poder conseguir fácilmente una gran variedad de acabados superficiales, con texturas similares a las de cualquier material de construcción.

7. Poder adicionar en el mortero, otros materia les tales como Perlita, Vermiculita o Cenizas Volantes, que unido a la presencia de las fin bras de vidrio generan productos de gran resistencia al fuego.

\section{Aplicaciones}

Una de las más interesantes y recientes aplicaciones del G.R.C. ha sido la remodelación llevada a cabo por Hispano-Alemana de Construcciones, Sociedad Anónima de la fachada y cierres frontales interior y exterior de la marquesina del Estadio Santiago Bernabéu del Real Madrid Club de Fútbol.

Esta importante obra planteaba grandes dificultades de diseño, planificación y construcción por tenerse que realizar las obras sin interrupción del uso del estadio durante un año.

Cinéndonos en sintesis a la fachada y cierres de marquesinas, las caracteristicas de estas unidades de obra eran:

- Plazo de fabricación ...................... 11 meses

- N. ${ }^{\circ}$ de piezas ............................... 6.264

- N. ${ }^{\circ}$ de unidades diferentes ............ 1.936

- Metros cuadrados de moldes ......... 800

- N. ${ }^{\circ}$ de moldes ........................... 136

- Metros cuadrados totales ...............16.000

- Sandwich de $8 \mathrm{~cm}$ de espesor ........ 4.000 $\mathrm{m}^{2}$

- Sandwich de $6 \mathrm{~cm}$ de espesor ........ $8.000 \mathrm{~m}^{2}$

- Elementos celosía $20 \times 10 \ldots \ldots \ldots . . . .1 .000 \mathrm{~m}^{2}$

- Lámina nervada .......................... $3.000 \mathrm{~m}^{2}$

- Dimensión máxima de panel: $12 \mathrm{~m}^{2}$ con un peso de $800 \mathrm{~kg}$.

- Metros lineales de junta: 18.000, que suponen una repercusión por metro cuadrado de panel de sólo 1,13 metros lineales.

- 100 tipos de anclajes diferentes.

Como indice de la complicación de esta obra diremos que el número de planos de definición de piezas realizadas por el equipo de diseño de prefabricado de Hispano-Alemana de Construcciones ascendió a la cifra de 2.077 .

La elección del G.R.C., frente a otros materiales, se basó, además de las ventajas ya reseñadas, en: 
- Posibllidad de revestir áreas grandes y conflic tivas.

- Ligereza en la manipulación y consecuente simplicidad de los elementos de anclaje, de especial importancia por tratarse de una estructura no construida especificamente para recibir un prefabricado, salvo en la marquesina, que era de nuevo diseño (Fig. 4).

Una vez aceptada la elección del G.R.C. como material de trabajo, se realizaron una serie de prototipos para definir: color, textura, formas de manipulación, fijación en obra y sistema de juntas.

Paralelamente se confeccionó un programa de trabajo para la fabricación de los diterentes modelos y tipos en función del programa general de la obra. Esto era importante, puesto que la obra tenia un proceso de demolición y nueva ejecución que no interrumpió la celebración de partidos.

En su momento, se planteó asimismo la alternativa del lugar de fabricación: in situ o taller aparte, posibilidades ambas factibles por la tecnología del G.R.C. En el primer caso se consiguia una reducción de costes al eliminar el transporte, pero im-

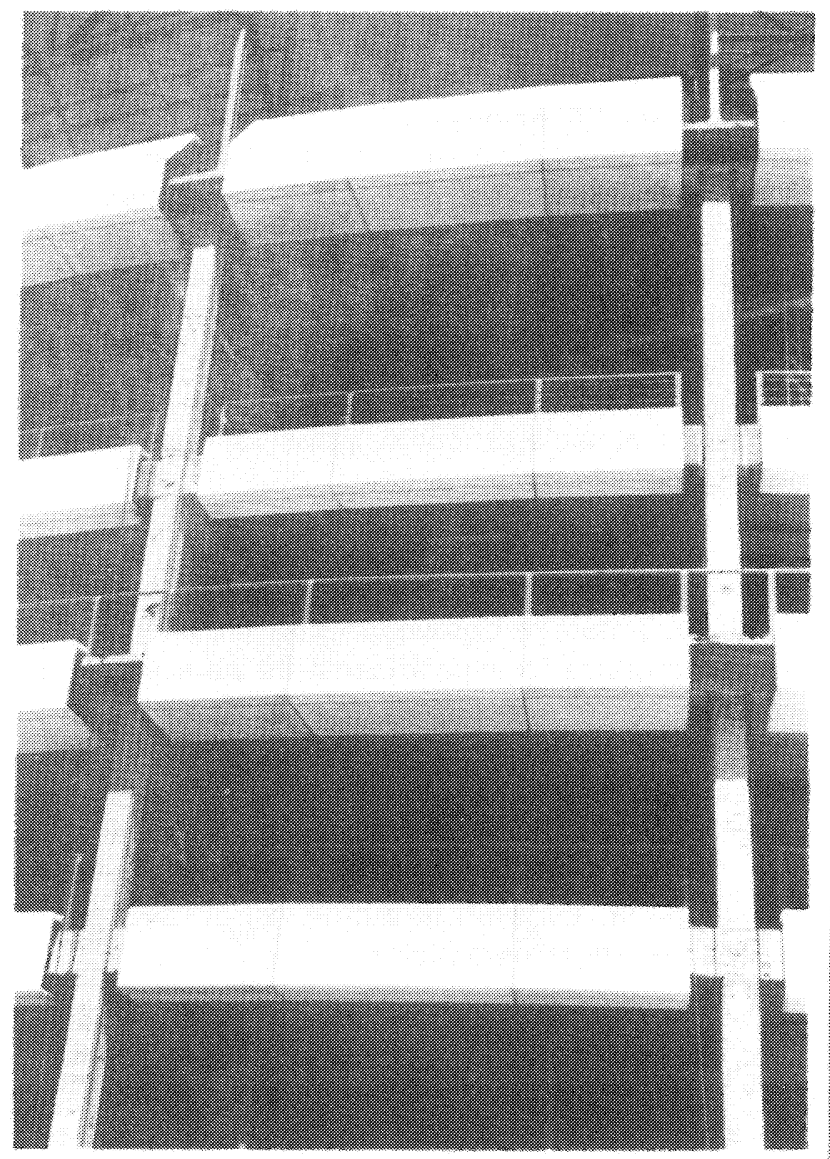

Fig. 4. -- Piezas sobre la antigua estructura del estadio.

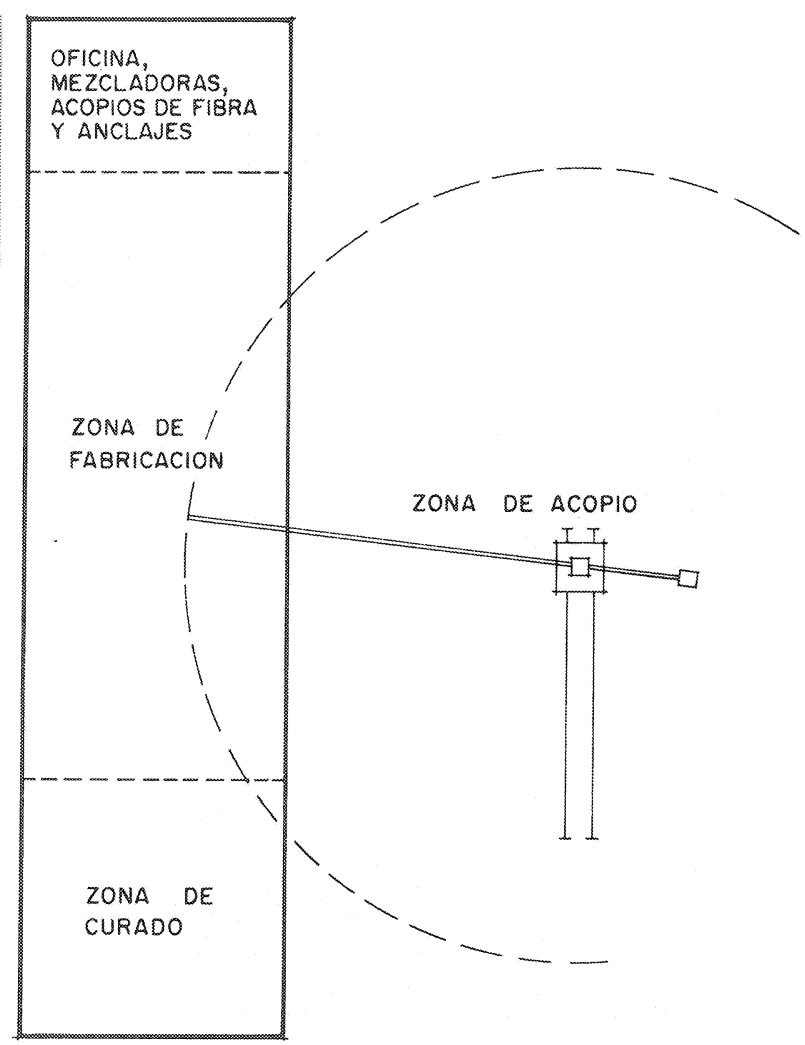

Fig. 5. - Esquema de taller del G.R.C.

plicaba más gastos de instalación. Se decidió la prefabricación en el taller de Hispano-Alemana de Construcciones, S. A., para un mejor control de calidad de los elementos al contar con una infraestructura más adecuada, en cuanto a medios técnicos, de curado, acopio, etc.

Esquemáticamente el taller se distribuye según el gráfico de la figura 5.

De la superficie total, como es habitual en este tipo de instalaciones, se destina un $15 \%$ a la infraestructura fija (preparación de mortero y hormigón de poliestireno) así como de acopios en general (fibra, anclaje, etc.); un $25 \%$ para zona de repasos y curado y el $60 \%$ restante es ocupado por los moldes y la vía de traslado de las máquinas proyectoras.

\section{Materiales}

Los materiales empleados en las diferentes piezas fueron por exigencias de proyecto: cemento blanco PB-450, arena seleccionada de Prádena (Sego via), agua y fibra de vidrio, Cem-Fil AR y el método tue de proyección manual. Figs. 6 y 7 .

Los materiales para el hormigón de poliestireno weron cemento gris $P_{-450}$ ARl y perlas de poliestireno. 


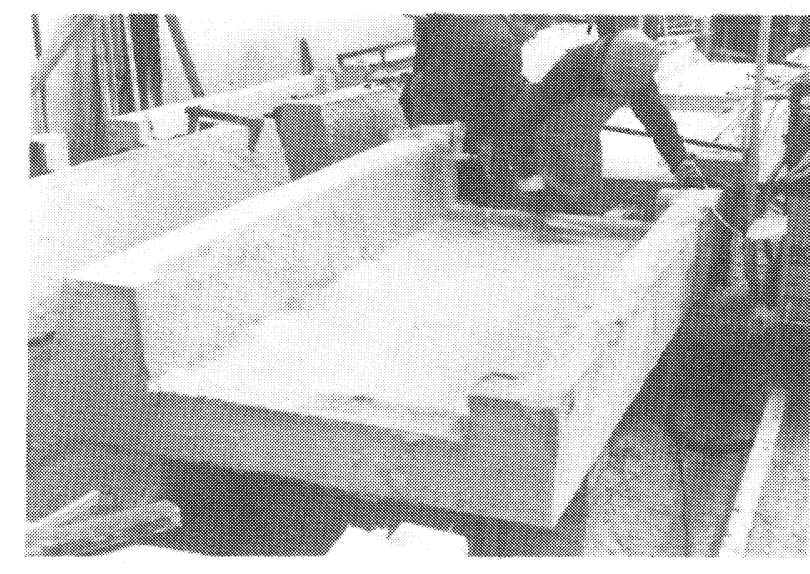

Fig. 6. - Compactado de la primera capa de G.R.C. proyectada sobre un molde del mismo material.

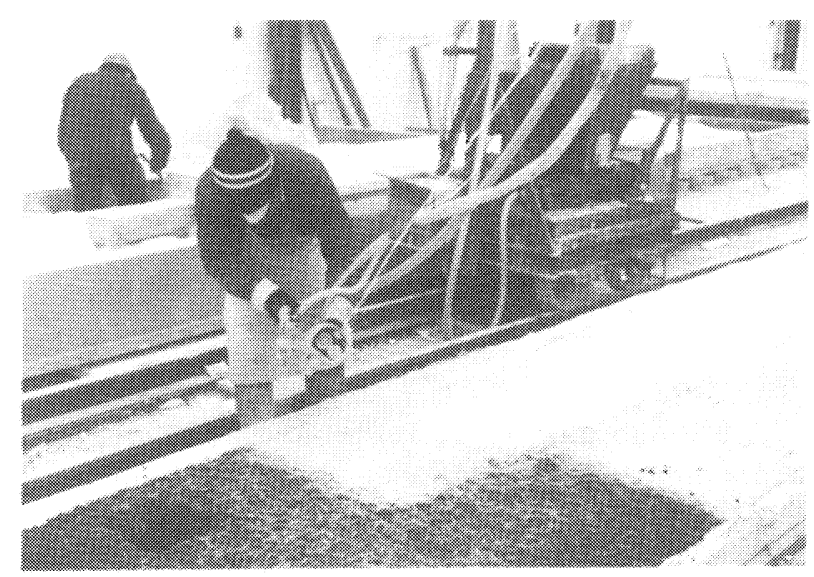

Fig. 7. - Proyección de la segunda capa de G.R.C. en formación de sandwich con hormigón de poliestireno.

El G.R.C. se dosificó en peso con las siguientes cantidades por amasada:

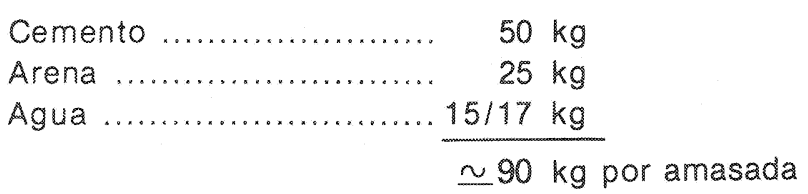

fibra Cem-FIL $5 \%$ en peso del compuesto en tresco

Relación agua/ cemento $=10,30 \sim 0,34)$

Y el hormigón de pollestireno:

Cemento ..........................5 $50 \mathrm{~kg}$

Poliestireno ..................... $3 \mathrm{~kg}$

Agua ........................ $17 \mathrm{~kg}$

$\simeq 70 \mathrm{~kg}$ por amasada

Los paneles sandwich de 8 o $6 \mathrm{~cm}$ lo formaban dos capas de 6 o $10 \mathrm{~mm}$ de G.R.C. que encerra- ban un núcleo de hormigón de poliestireno de 6 ó $4 \mathrm{~cm}$ respectivamente. Los anclajes se embebian en núcleos macizos de G.R.C.

\section{Montaje}

Los equipos de montaje en obra se formaban con: un soldador, un gruista, un manipulador en zona de acopio y dos en el lugar de colocación para aplomado, nivelación y alineación.

Se necesitaron, dada la diversidad de la geometria de las piezas, la altura y posición relativa donde éstas se colocaban, siete útiles equilibradores, que permitieron alcanzar aquellas zonas donde no existía posibilidad de colocación directa con las grúas. El mayor útil empleado en el montaje de la zona inclinada en el exterior de la marquesina tenía unas dimensiones de $1.75 \mathrm{~m}$ de ancho, $5 \mathrm{~m}$ de largo y $7,20 \mathrm{~m}$ de altura, con un peso de $1.000 \mathrm{~kg}$.

También, dada la ausencia de forjados, fue necesaria la utilización de una plataforma de trabajo móvil.

En función de las caracteristicas de las piezas se emplearon grúas torres automontables sobre neumáticos con un alcance en punta de $20 \mathrm{~m}$, una altura bajo gancho de $24 \mathrm{~m}$ y una carga en punta de $750 \mathrm{~kg}$, otras montadas sobre carriles con carretón adaptable a la pendiente de cada tra mo de calle, con un alcance en punta de $45 \mathrm{~m}$, una altura bajo gancho de $40 \mathrm{~m}$ y una carga en punta de $2.000 \mathrm{~kg}$, dotadas de doble botonera para su accionamiento desde el apllado o el montaje. Los elementos especiales como celosias, etc. se izaron con grúa móvil telescópica. (Fig. 8).

Tanto las dimensiones de las piezas como los da tos de replanteo se obtuvieron con un microprocesador, programado especificamente para este proyecto, utilizándose como datos la distancia entre ejes de pilares, el radio teórico de la zona considerada calculado previamente, y los datos de las sección transversal de la pieza en cuestión. Esto unido a la excelente labor y dedicación del equipo de topografia, permitió la definición de piezas, in cluso antes de la demolición.

Dado el bajo peso de las piezas, el transporte, desde el taller hasta la obra se efectuó con cam mión-grúa, lo que permitió independizar las labores de carga y descarga (Fig. 9). 


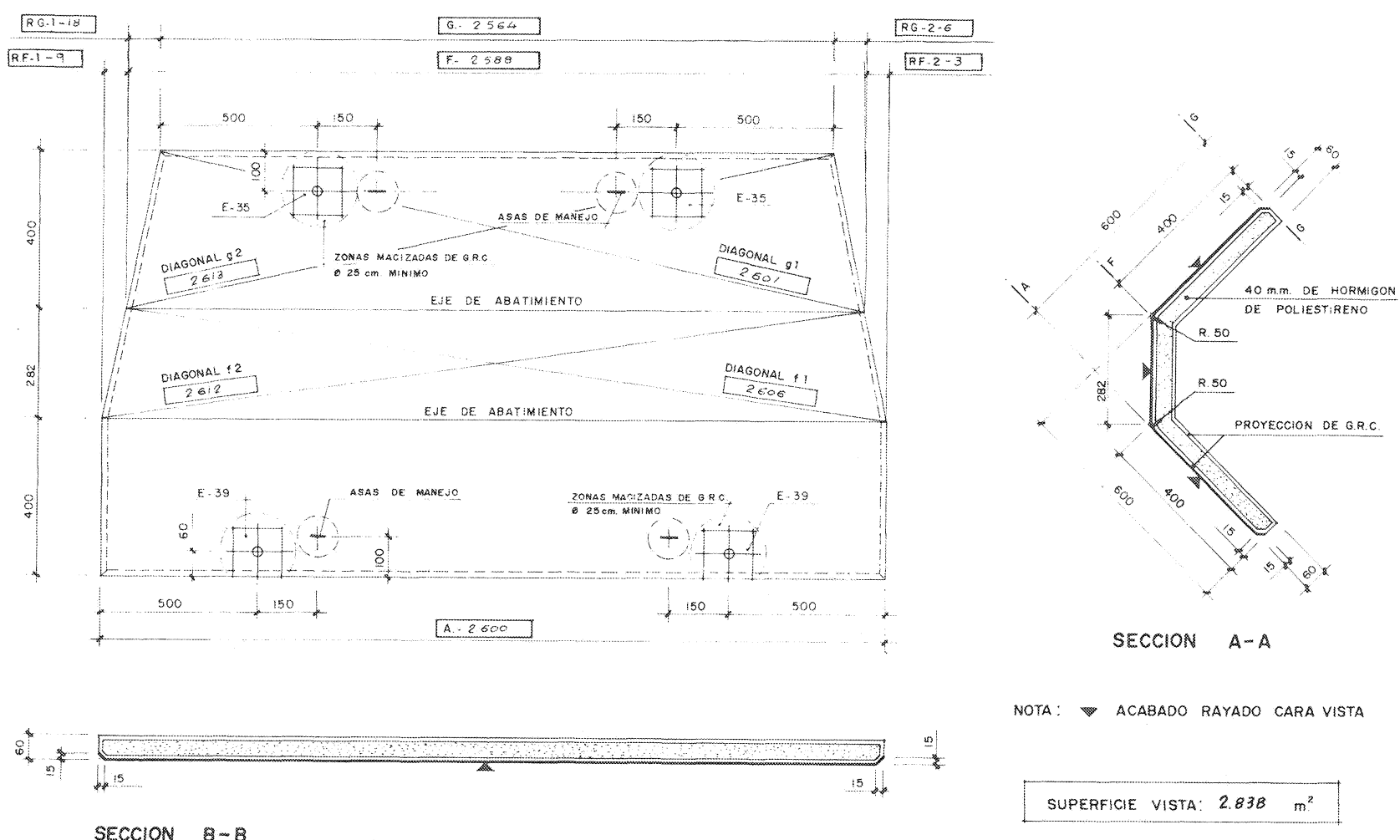

Fig. 8.-Definición de pieza.

\section{Acabados}

En cuanto a los acabados se eligió una textura rayada en las partes cuyo desmoldeo lo permitia (zonas frontales de parementos) que aminoró sombras o brillos que siempre se producen por diferencias en la coloración de los cementos, planicidad de los moldes, y acumulaciones de desmoldeante. El acuerdo curvo de las zonas horizontales con las verticales, que impidió utilizar laterales abatibles, se acabó con textura lisa.

Las superficies de las piezas se trataron con una impregnación al silicato y silicón que repele la absorción del agua y permite el paso de vapor a través de la pieza (a fin de evitar la posibilidad de condensación en el núcleo del sandwich). Dicha pelicula transparente no aporta brillos ni tonalidades a las superficies de los paramentos. En las zonas de difícll acceso las piezas fueron tratadas previamente en taller.

La unión entre piezas, y entre éstas y los elementos de remate, se solucionó a base de una junta ahuecada de una sóla fase. El sellado se realizó con masilla de estanquidad de polisulfuro de dos componentes, que permite un movimiento de junta $+20 \%$ y presenta una buena resistencia a la intemperie, a los rayos ultravioletas y abrasión.
Dicha masilla se moldeó in situ sobre un fondo de junta, formado por una barra premoldeada de poliuretano elástico de celda cerrada. Se utilizó además una imprimación adherente-deslizante en el perímetro de contacto. En las juntas de dilata-

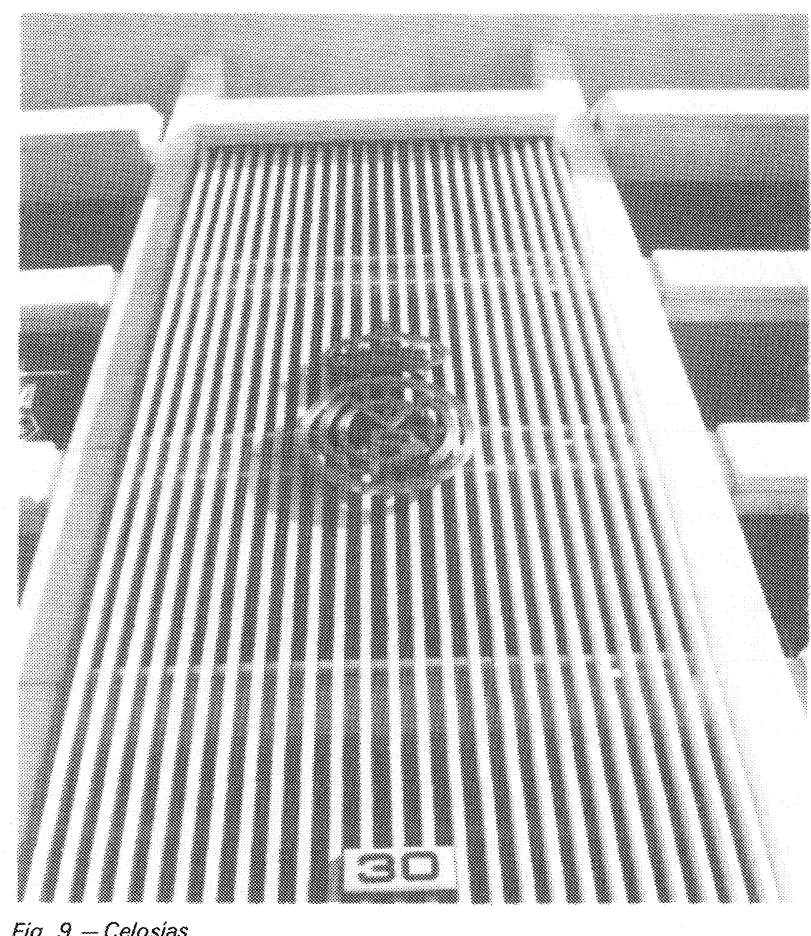


ción del edificio se aumentó el ancho a fin de que el sellado se acomodara al mayor movimiento.

\section{Moldes}

El criterio fue: madera para los modelos de los que posteriormente se obtendrian los moldes de G.R.C.; acero en los casos en que fue necesario laterales móviles y con un número elevado de puestas; y escayola en los casos de utilizaciones complicadas y bajo número de puestas.

La textura rayada se obtuvo con un entelado vinílico de los modelos de madera.

La durabilidad del molde se aumentó con la aplicación de un barniz en su superficie. El desmoldeo se facilitó mediante la utilización de agentes desmoldeantes.

El proceso de desmoldeo, una vez iniciada la fabricación, se realizó a las 16-24 horas. El curado se aceleró ocasionalmente con aportación de calor.

En todos los casos se cuidó el dimensionado y geometria de los moldes para permitir la libre re tracción de las piezas.

\section{Anclajes}

Debido a la diversidad de formas y posiciones de las piezas se utilizaron: asas de desmoldeo, manguitos roscados de métrica 12 y 16 en manejo, desmoldeo o fijación, placas de anclaje de diversos tipos, dotadas de hembras roscadas interiores, tacones de apoyo con placa de asiento y tam ladros pasantes de manejo. Todos estos elementos se fundian en zonas macizas de G.R.C. de diferentes dimensiones en función de la carga a soportar.

Los anclajes in situ y su fijación a las piezas se estudiaron de forma que permitieran regular las tolerancias de alineación y nivel de la estructura existente y además, una vez montados, una corrección final de las tolerancias de montaje y fam bricación absorbiendo los cambios producidos por la temperatura y humedad. Los elementos se fijaban a la estructura por soldadura, tacos de expansión, resinas de adherencia o pernos.

Las tolerancias admisibles en el montaje para las plezas de mayor tamaño se estimaron en nivelación $+2 \mathrm{~cm}$, aplomado $+1 \mathrm{~cm}$ y desplazamiento en la alineación $+2 \mathrm{~cm}$.
Los anclajes llevan un tratamiento antioxidante por galvanizado o resinas epoxidicas, repasándo se dicho tratamiento una vez montadas las piezas.

\section{Control de calidad}

Se centró en tres aspectos fundamentalmente: materias primas, producción y elaborados.

En el primer caso se aplicó la norma EH-80 (EH-73 en vigor en la redacción del proyecto).

El control del proceso de producción constó de:

- Determinación de la viscosidad del mortero.

- Comprobaciones de los caudales de mortero y fibra.

- Comprobaciones de contenido de fibra sobre G.R.C. tresco.

- Preparación de probetas, que ensayadas a flexión deberian cumplir los valores característicos mínimos a 28 dias:

L.O.P. (limite elástico)

$6,5 \mathrm{~N} / \mathrm{mm}^{2}$

M.O.R. (tensión de rotura) $21,0 \mathrm{~N} / \mathrm{mm}^{2}$

\section{Análisis y recapitulación}

- En la ejecución de la obra se comprobó una superación de las tolerancias de la estructura a cubrir con piezas prefabricadas. Hubo nece sidad de refuerzo en algunas zonas y modificación de anclajes por aparición de geometrias no previstas.

- Como consecuencia del replanteo manual diario en el taller se observó superación de algunas tolerancias de los anclajes y piezas.

- Aparecieron diferentes matices de color en las piezas en función de la época de fabricación. Estos matices fueron desapareciendo con el transcurso del tiempo y la actuación de los rayos solares. Aún no hay tiempo para comprobar la influencia de la contaminación ambiental.

\section{Epílogo}

En la exposición anterior se han descrito breve mente las propiedades y caracteristicas del G.R.C. y su papel en una importante realización. 
Informes de la Construcción

Actualmente existen unos 500 concesionarios que utilizan la fibra Cem-FIL bajo licencia y se cuenta con realizaciones en todo el mundo, en campos tan diversos como:

- Construcción masiva de vivienda social.

- Construcciones hidráulicas.

- Señalización urbana y mobillario exterior.

- Elementos antifuego.

- Encotrados perdidos.

Resulta sin embargo muy dificil acotar los limites de utilización de este material, ya que en los tres congresos mundiales celebrados:

- Brighton, 1977

- Londres, 1979,

- Paris, 1981,

se han estudiado aplicaciones del G.R.C. reservadas tradicionalmente a materiales tales como la madera, aluminio y fundición.

\section{Bibliografía}

- ALLEN. H.G. - Stifness and Strength of Two Glass Fibre Reinforced Cement Laminates. Joumal of Composite Materials Vol. 5, April 1971. pp 194-204.

- ALLEN, H.G. - The Purpose and Methods of Fibre Reinforce ment. Prospects for Fibre Reinforced Construction Materials Proceedings of the International Bulding Exhibition Conference. Olimpya, London 24th - November 1974 op 3-14 (D.o.E.).

- PROCTOR, B.A. - Fibre Reinforced Composite Materials. Faraday Special Discussions of the Chemical Society. $N \cdot{ }^{\circ}$ 2. $1972 \mathrm{pp} 63-76$

- MAJUMDAR, A. J. and NURSE, R.W. - Glass Fibre Reinforced Cement Materials Science and Engineering. N. ${ }^{\circ} 15.1974$. pp 107-127.

- OAKLEY, D.R. and PROCTOR, B.A.- Tensile Stress Strain Behaviour of Glass Fibre Reinforced Cement Composites in Neville A. (Editor). Proceedings of the RLEM Symposium on Fibre Reinforced Cement and Concrete. The Construction Press Limited. 1975. 650 pp. See esp po. 347-359.

- Quality Control Test Booklet. Application Data for Use with Cem Fll Fibre. Pilkington Brothers Ltd. St. Helens, Merseyside. England IOn issue to all Licensees: available to G.R.C. Specifiers on request).

- Al, M.A.; MAJUMDAR, A.J. and SINGH, B. - The Properties of Glass Fibre Cement - The Effect of Fibre Length and Content, Journal of Materials Science. Vol 10 1975. pp $1732-1740$.

- A Study of the Properties of Cem-FIL OPC Composites, Bullding Research Establishment Current Paper CP $38 / 76$ Watford. Department of Environment 1976. $14 \mathrm{pp}$.

\section{publicación del i.e.t. t.c.c.}

\section{alojamiento y tecnología: ¿industrialización abierta?}

\section{Julián Salas Serrano}

Ingeniero Industrial

La experiencia nacional en construcción industrializada en la última década, aunque no abundante, puede resultar paralizante. Como reacción, este trabajo trata de elaborar y ordenar la información que, pegado al terreno, se ha acumulado durante los sesenta.

Auscultando tendencias avanzadas en otros paises y apoyandose en nuestra realidad cotidiana, el autor de este trabajo apuesta por la industrialización, presintiendo un futuro con pocos puntos en común con lo que en general, hasta bien reciente, se ha conocido como construcción industrializada.

Se abre el trabajo con un prólogo del Profesor Ciribini en el cual, con rigor conceptual y desde su rica experiencia, analiza algunos de los conceptos más polemicos del fenómeno de industrialización del sector construcción. El autor recopila un conjunto de aportaciones de maestros de la arquitectura al lento proceso de evolución tecnológica y conceptual, continuando con un intento de respuesta realista a la interrogante que flota en el sector nacional: ¿réquiem por la industrialización?

Se dedican otros capitulos a revisar to realizado y a encarar el futuro inmediato: el concepto de componente, su repercusión económica, la dificultad del proyecto a base de productos industriales, la nueva vigencia embrionaria de la construcción por medio de catálogos, los conceptos de hexibilidad e Frpecial atención dedica este trabajo a la exposición de lo que podiamos llamar nuevos derrón y pragmática de los enfoques más implantados en Europa.

Las siempre problemáticas interrelaciones entre normativa, calidad e industria se detallan de forma documentada y realista. Finaliza el trabajo con unas reflexiones dirigidas á ámbito empresarial y un esbozo de ureglas de juegon que faciliten al subsector la salida del kimpasse» actual.

Un volumen encuadernado en rústica, de $24 \times 16,5 \mathrm{~cm}$, compuesto de 160 páginas, con 109 figuras, 19 tablas y 86 referencias bibliográticas. Madrid, junio, 1981. 